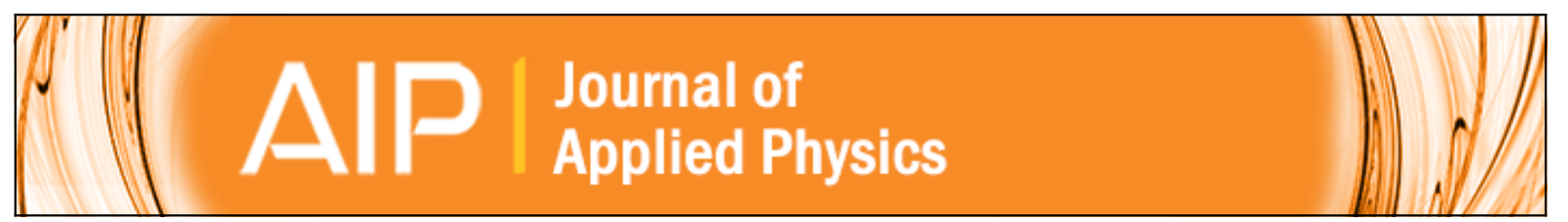

\title{
Electrical and piezoelectric properties of BiFeO3 thin films grown on SrxCa1-xRuO3- buffered SrTiO3 substrates
}

Yingbang Yao, Long Chen, Zhihong Wang, Husam Alshareef, and X. X. Zhang

Citation: Journal of Applied Physics 111, 114102 (2012); doi: 10.1063/1.4724332

View online: http://dx.doi.org/10.1063/1.4724332

View Table of Contents: http://scitation.aip.org/content/aip/journal/jap/111/11?ver=pdfcov

Published by the AIP Publishing

\section{Articles you may be interested in}

Significantly enhanced ferroelectricity and magnetic properties in ( $\mathrm{Sr} 0.5 \mathrm{Ca} 0.5) \mathrm{TiO} 3$-modified $\mathrm{BiFeO} 3$ ceramics J. Appl. Phys. 117, 174101 (2015); 10.1063/1.4919705

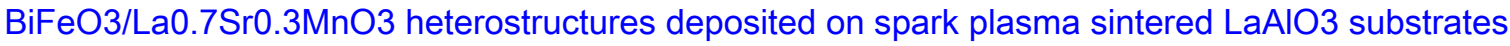
Appl. Phys. Lett. 104, 082914 (2014); 10.1063/1.4867021

Magnetic and structural properties of $\mathrm{BiFeO} 3$ thin films grown epitaxially on $\mathrm{SrTiO} / \mathrm{Si}$ substrates J. Appl. Phys. 113, 17D919 (2013); 10.1063/1.4796150

Multifunctional behavior of $\mathrm{ZnO}$ supported $\mathrm{Bi} 1-\mathrm{xDyxFeO} 3$ nanorods

J. Appl. Phys. 110, 054313 (2011); 10.1063/1.3636274

Effects of SrTiO3/TiO2 buffer layer on structural and electrical properties of BiFeO3 thin films grown on GaN (0002)

J. Appl. Phys. 109, 104108 (2011); 10.1063/1.3585836

MIT LINCOLN

LABORATORY CAREERS

Discover the satisfaction of innovation and service

to the nation
- Space Control

- Air \& Missile Defense

- Communications Systems \& Cyber Security

- Intelligence, Surveillance and

Reconnaissance Systems

- Advanced
Electronics
- Tactical Systems
" Homeland
Protection
- Air Traffic Control

LINCOLN LABORATORY

MASSACHUSETTS INSTITUTE OF TeCHNOLOGY

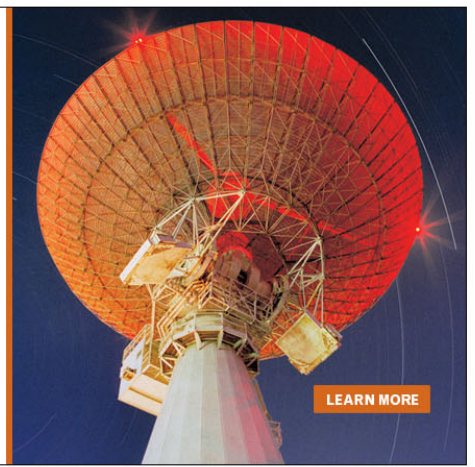




\title{
Electrical and piezoelectric properties of $\mathrm{BiFeO}_{3}$ thin films grown on $\mathrm{Sr}_{x} \mathrm{Ca}_{1-x} \mathrm{RuO}_{3}$-buffered $\mathrm{SrTiO}_{3}$ substrates
}

\author{
Yingbang Yao, ${ }^{1}$ Long Chen, ${ }^{1}$ Zhihong Wang, ${ }^{1}$ Husam Alshareef ${ }^{2}$ and X. X. Zhang ${ }^{1, a)}$ \\ ${ }^{1}$ Thin Film, Advanced Nanofabrication, Imaging \& Characterization Core Lab, King Abdullah University \\ of Science and Technology, Thuwal, 23955-6900 Jeddah, Saudi Arabia \\ ${ }^{2}$ Materials Sciences and Engineering, King Abdullah University of Science and Technology, \\ Thuwal, 23955-6900 Jeddah, Saudi Arabia
}

(Received 11 February 2012; accepted 2 May 2012; published online 1 June 2012)

\begin{abstract}
(001)-oriented $\mathrm{BiFeO}_{3}(\mathrm{BFO})$ thin films were grown on $\mathrm{Sr}_{\mathrm{x}} \mathrm{Ca}_{1-\mathrm{x}} \mathrm{RuO}_{3^{-}}(\mathrm{SCRO} ; \mathrm{x}=1,0.67,0.33,0)$ buffered $\mathrm{SrTiO}_{3}$ (001) substrates using pulsed laser deposition. The microstructural, electrical, ferroelectric, and piezoelectric properties of the thin films were considerably affected by the buffer layers. The interface between the BFO films and the SCRO-buffer layer was found to play a dominant role in determining the electrical and piezoelectric behaviors of the films. We found that films grown on $\mathrm{SrRuO}_{3}$-buffer layers exhibited minimal electrical leakage while films grown on $\mathrm{Sr}_{0.33} \mathrm{Ca}_{0.67} \mathrm{RuO}_{3}$-buffer layers had the largest piezoelectric response. The origin of this difference is discussed. (c) 2012 American Institute of Physics. [http://dx.doi.org/10.1063/1.4724332]
\end{abstract}

\section{INTRODUCTION}

Bismuth ferrite $\left(\mathrm{BiFeO}_{3}\right)$ has been extensively studied due to its unique multiferroic properties, which are promising for applications in novel magnetoelectric (ME) devices. ${ }^{1,2}$ Furthermore, promising photovoltaic performances, such as large open-circuit voltage, ${ }^{3}$ high quantum efficiency, ${ }^{4}$ and appreciable response to visible lights. ${ }^{5}$ Domain walls and interfaces (grain boundaries and ferroelectric-electrode interfaces) are found to play important roles in the photovoltaic effects. ${ }^{3,6,7}$ BFO is ferroelectric (FE) and antiferromagnetic (Anti-FM) in bulk samples, whereas it is FE and ferromagnetic (FM) in thin films and nanoparticles. ${ }^{1-10}$ Moreover, the FE Curie temperature and Anti-FM Neel temperature of BFO are $810^{\circ} \mathrm{C}$ and $380^{\circ} \mathrm{C}$, respectively, which are high enough for normal applications. ${ }^{9,10}$ The appearance of FM behavior in BFO thin films is related to residual strain, which deforms the crystallographic structure from rhombohedral to tetragonal to some extent. ${ }^{1}$ Such deformation suppresses the AntiFM spiral spin structure and results in the appearance of FM (Ref. 11) and leads to a large electrical remnant polarization $\left.\left(\sim 130 \mu \mathrm{C} / \mathrm{cm}^{2}\right)\right)^{1,12}$ Recently, a substantial piezoelectric response $(\sim 5 \%)$ was observed in BFO films with coexistence of tetragonal and rhombohedral crystal structures. ${ }^{13}$ Additionally, transitions between the tetragonal phase and the rhombohedral phase can be controlled by external electrical field ${ }^{14}$ and a new temperature-induced phase transitions around $100^{\circ} \mathrm{C}$ was recently observed in these highly strained BFO films. ${ }^{15}$ It seems clear, then that strain considerably affects the magnetic, electrical and piezoelectric behaviors of BFO films. Here, we report on how the introduction of different strain states affects BFO thin films grown on different substrates. To this end, we chose $\mathrm{Sr}_{\mathrm{x}} \mathrm{Ca}_{1-\mathrm{x}} \mathrm{RuO}_{3^{-}}(\mathrm{SCRO} ; \mathrm{x}=1$, $0.67,0.33,0)$ buffered $\mathrm{SrTiO}_{3}(001)$ crystals as the substrates. SCRO is highly conductive and is often used as the bottom

\footnotetext{
a) Author to whom correspondence should be addressed. Electronic mail: xixiang.zhang@kaust.edu.sa.
}

electrode in electrical characterizations. ${ }^{1,12-14,16,17}$ The lattice constants of pseudo-cubic SCRO are $3.92 \AA$, $3.89 \AA$, $3.87 \AA$, and $3.84 \AA$, for $\mathrm{x}=1,0.67,0.33$, and 0 , respectively (as shown below). The lattice constant of bulk BFO is $3.96 \AA$, which is clearly larger than that of SCRO. Therefore, the expected lattice mismatch would be $1.1 \%, 1.9 \%, 2.3 \%$, and $3.0 \%$, respectively. In addition, the expected stress state would be compressive. Although previous work used thin $\mathrm{SrRuO}_{3}$-buffered layers (10-80 nm thick) as the bottom electrode in BFO films and varied the strain state by using different substrates, such as $\mathrm{SrTiO}_{3}, \mathrm{LaAlO}_{3}, \mathrm{YAlO}_{3}$, and $\mathrm{Pt},{ }^{18-21}$ the effects of conductive buffer layers on the performance of BFO thin films has not yet been studied systematically.

\section{EXPERIMENTAL}

SCRO and BFO targets were synthesized using the conventional solid-state reaction technique. $\mathrm{BiFeO}_{3}$ ceramics were prepared from reagent-grade $\mathrm{Bi}_{2} \mathrm{O}_{3}$ and $\mathrm{Fe}_{2} \mathrm{O}_{3}$ powders (Sigma-Aldrich). The raw materials were well mixed by ball-milling, pressed into 1-in.-diameter disks under a uniaxial pressure of $200 \mathrm{MPa}$, and then sintered at $795^{\circ} \mathrm{C}$ for 30 min. Excess $\mathrm{Bi}$ was added to compensate for the loss of $\mathrm{Bi}$ during processing. The $\mathrm{Sr}_{\mathrm{x}} \mathrm{Ca}_{1-\mathrm{x}} \mathrm{RuO}_{3}$ targets were prepared using $\mathrm{SrCO}_{3}, \mathrm{CaCO}_{3}$, and $\mathrm{RuO}_{2}$ raw powders, which were sintered at $1500{ }^{\circ} \mathrm{C}$ for $4 \mathrm{~h}$. The target composition and crystal structure were confirmed by $\mathrm{x}$-ray fluorescence (XRF) and $\mathrm{x}$-ray diffraction (XRD) patterns.

The SCRO-buffer layers and BFO films were successively deposited onto $\mathrm{SrTiO}_{3}$ (001) single-crystal substrates using pulsed laser deposition (PLD). During deposition, the substrate temperature was fixed at $750^{\circ} \mathrm{C}$ for SCRO and $650^{\circ} \mathrm{C}$ for $\mathrm{BFO}$, and the oxygen pressure was kept at 150 mTorr for both layers. The thicknesses of the SCRO layers and BFO layers were both around $380 \mathrm{~nm}$. The laser $(\mathrm{KrF}$, $248 \mathrm{~nm}$ ) was operated at $5 \mathrm{~Hz}$ with an energy density of $7 \mathrm{~J} / \mathrm{cm}^{2}$ at the target surface. The distance between the target and substrate was $4 \mathrm{~cm}$. After deposition, 400 Torr of oxygen was 
introduced into the chamber and the sample was annealed at $650{ }^{\circ} \mathrm{C}$ for $20 \mathrm{~min}$ under this oxygen environment. Au electrodes of that were $20 \mathrm{~nm}$ thick and $0.2 \mathrm{~mm}$ in diameter were sputtered onto the BFO films. The crystal structure of the samples was characterized by an x-ray diffractometer (XRD, Bruker D8 Discover). The surface morphology and piezoelectric response were characterized by atomic force microscopy (AFM, Agilent 5400). Electrical leakage currents and ferroelectric polarization were measured using a ferroelectric tester (aixACCT).

\section{RESULTS AND DISCUSSION}

The XRD patterns of the BFO thin films and the SCRObuffer layers are shown in Figure 1. The SCRO-buffer layers were well crystallized and oriented in the (001) direction. The BFO films grown on $\mathrm{SrRuO}_{3}$ - and $\mathrm{Sr}_{0.67} \mathrm{Ca}_{0.33} \mathrm{RuO}_{3}$-buffered substrates exhibited only (001) orientations. In the BFO films grown on $\mathrm{Sr}_{0.33} \mathrm{Ca}_{0.67} \mathrm{RuO}_{3^{-}}$and $\mathrm{CaRuO}_{3}$-buffered substrates, a weak (110) peak, around $10 \%$ of the intensity of the major (001) peak, also appeared, as shown in Figure 1(a).The larger lattice mismatch between the BFO films and these two buffered layers is the likely reason for this difference. In all the films, tiny amounts of secondary-phase $\mathrm{Bi}_{25} \mathrm{FeO}_{40}$ and
$\mathrm{Bi}_{2} \mathrm{Fe}_{4} \mathrm{O}_{9}$ were detected. Decomposition of the BFO film during high-temperature processing could be the origin of the phase impurities. $^{22-24}$ Enlarged views of the (001) peaks are shown in Figures 1(c) and 1(d) for BFO films and the buffer layers, respectively. The (001) diffraction positions $(2 \theta)$ of the buffer layers were $22.51^{\circ}, 22.58^{\circ}, 22.63^{\circ}$, and $23.12^{\circ}$, for $\mathrm{SrRuO}_{3}, \mathrm{Sr}_{0.67} \mathrm{Ca}_{0.33} \mathrm{RuO}_{3}, \mathrm{Sr}_{0.33} \mathrm{Ca}_{0.67} \mathrm{RuO}_{3}$, and $\mathrm{CaRuO}_{3}$, respectively. The corresponding bulk values were $22.68^{\circ}$, $22.86^{\circ}, 22.96^{\circ}$, and $23.13^{\circ}$, as shown in the inset of Figure 1(d). Low shifts of XRD peak positions (or increase of lattice constant) were present in all buffer layers, indicating tensile stress in the SCRO layers. In the BFO films, the (001) peak positions were $22.42^{\circ}, 22.44^{\circ}, 22.48^{\circ}$, and $22.50^{\circ}$, for buffer layers of $\mathrm{SrRuO}_{3}, \mathrm{Sr}_{0.67} \mathrm{Ca}_{0.33} \mathrm{RuO}_{3}, \mathrm{Sr}_{0.33} \mathrm{Ca}_{0.67} \mathrm{RuO}_{3}$, and $\mathrm{CaRuO}_{3}$, respectively. Meanwhile, the BFO target had its (001) peak at $22.40^{\circ}$. In comparison with its bulk value, the lattice constant of BFO film decreased gradually with different SCRO buffer layers from $\mathrm{SrRuO}_{3}$ to $\mathrm{CaRuO}_{3}$. Therefore, the stress state (compressive) of the BFO films increased gradually as the buffer layer changed from $\mathrm{SrRuO}_{3}$ to $\mathrm{CaRuO}_{3}$.

AFM images showing the surface microstructures of the BFO films are presented in Figure 2. All the samples are dense, uniform with no large pores. The grain sizes were
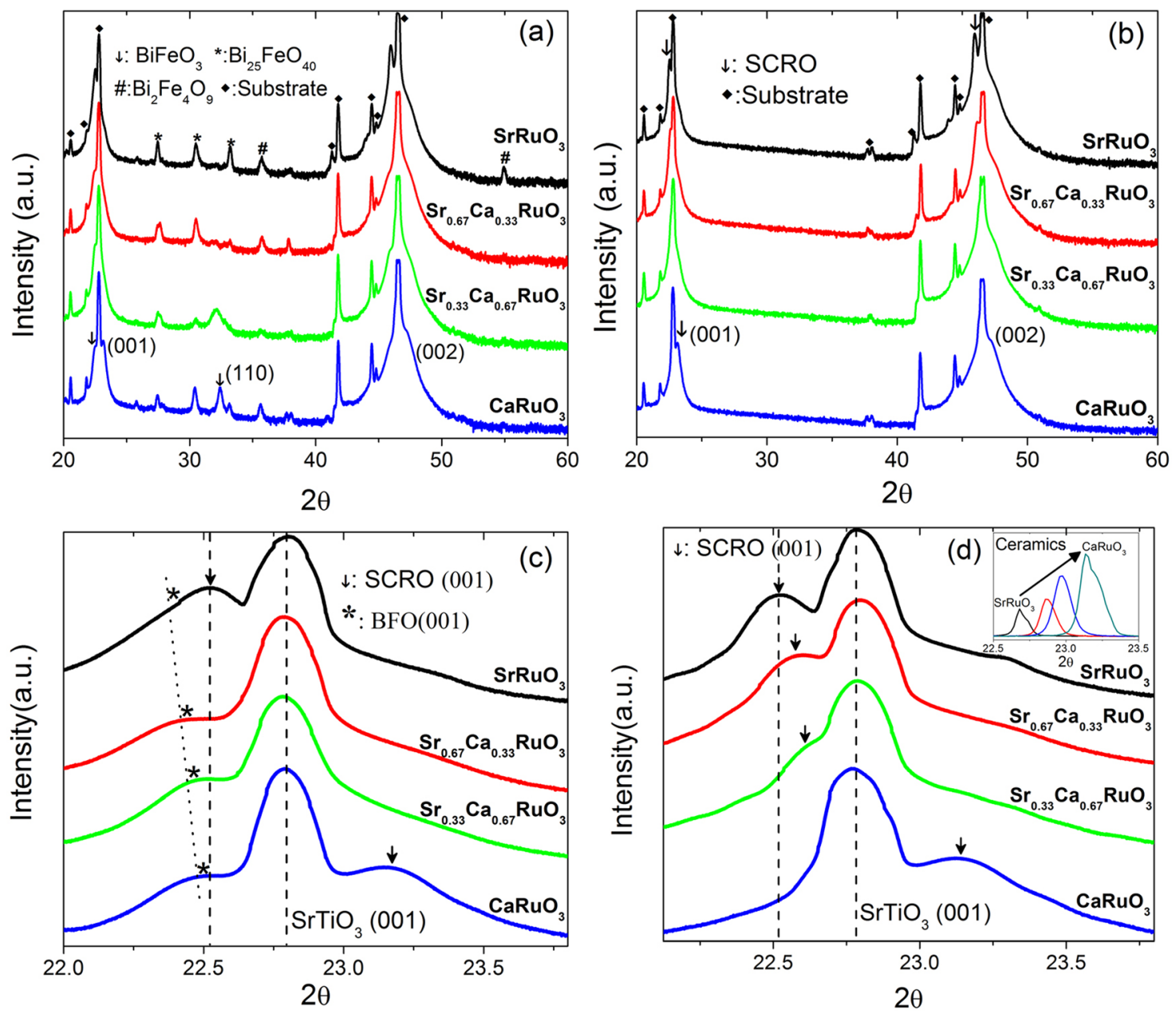

FIG. 1. XRD patterns of (a) BFO thin films on $\mathrm{Sr}_{\mathrm{x}} \mathrm{Ca}_{1-\mathrm{x}} \mathrm{RuO}_{3}$-buffered $\mathrm{SrTiO}_{3}$ substrates, (b) bare $\mathrm{Sr}_{\mathrm{x}} \mathrm{Ca}_{1-\mathrm{x}} \mathrm{RuO}_{3}$-buffered $\mathrm{SrTiO}_{3}$ substrates, and enlarged view of the pseudo-cubic (001) diffraction peaks of (c) BFO thin films and (d) $\mathrm{Sr}_{\mathrm{x}} \mathrm{Ca}_{1-\mathrm{x}} \mathrm{RuO}_{3}$-buffer layers. Inset in (d) shows the (001) diffraction peaks of bulk $\mathrm{Sr}_{\mathrm{x}} \mathrm{Ca}_{1-\mathrm{x}} \mathrm{RuO}_{3}$ ceramics. The dotted lines are guides. 

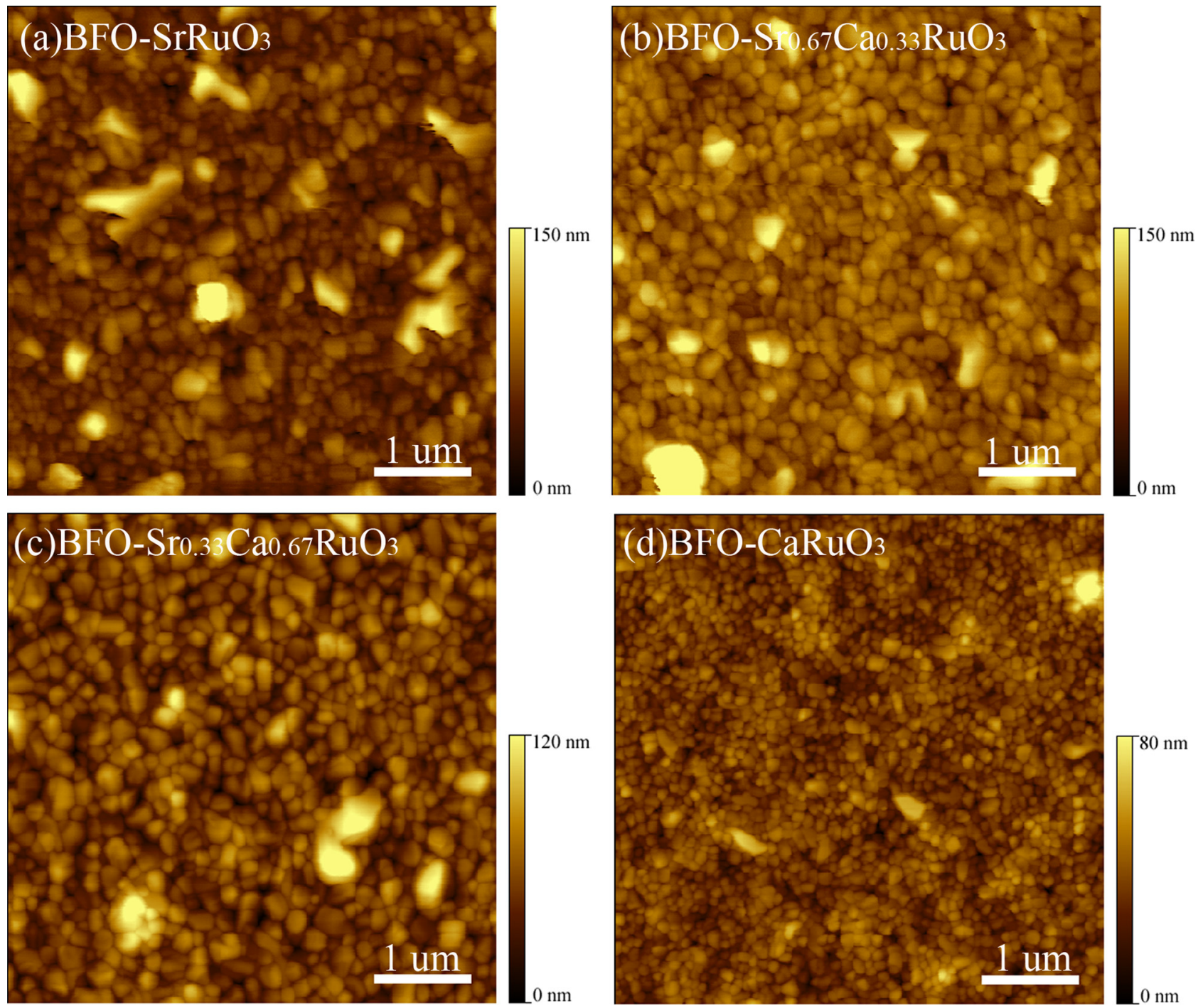

FIG. 2. AFM images of (a) BFO-SrRuO 3 , (b) BFO-Sr $\mathrm{Br}_{0.67} \mathrm{Ca}_{0.33} \mathrm{RuO}_{3}$, (c) $\mathrm{BFO}-\mathrm{Sr}_{0.33} \mathrm{Ca}_{0.67} \mathrm{RuO}_{3}$, and (d) $\mathrm{BFO}-\mathrm{CaRuO}_{3}$.

$260 \mathrm{~nm}, 207 \mathrm{~nm}, 186 \mathrm{~nm}$, and $134 \mathrm{~nm}$ in films with $\mathrm{SrRuO}_{3}$, $\mathrm{Sr}_{0.67} \mathrm{Ca}_{0.33} \mathrm{RuO}_{3}, \quad \mathrm{Sr}_{0.33} \mathrm{Ca}_{0.67} \mathrm{RuO}_{3}$, and $\mathrm{CaRuO}_{3}$-buffer layers, respectively. The corresponding surface roughness $\left(\mathrm{R}_{\mathrm{a}}\right)$ values were $15.8 \mathrm{~nm}, 14.6 \mathrm{~nm}, 12.1 \mathrm{~nm}$, and $7.0 \mathrm{~nm}$, respectively. The BFO film grown on the $\mathrm{SrRuO}_{3}$-buffered layer had the largest grain size and also the most rough surface, while the smallest grain size and the smoothest surface were found in the film with the $\mathrm{CaRuO}_{3}$-buffer layer. The smaller grains, or more grain boundaries, observed in the $\mathrm{BFO}$ on the $\mathrm{CaRuO}_{3}$-buffer layer can be understood in terms of film energy. ${ }^{25}$ In largely strained and thick films (thicker than tens of nanometers), grain boundaries are energetically favorable to reduce the strain energy. This will result in smaller grains. Another possible reason for the difference in BFO grain sizes may be associated with the microstructures of the underlying buffered layers, which are shown in Figure 3. The roughness values $\left(\mathrm{R}_{\mathrm{a}}\right)$ were $1.79 \mathrm{~nm}, 0.52 \mathrm{~nm}, 0.65 \mathrm{~nm}$, and $1.30 \mathrm{~nm}$ for $\mathrm{SrRuO}_{3}, \mathrm{Sr}_{0.67} \mathrm{Ca}_{0.33} \mathrm{RuO}_{3}, \mathrm{Sr}_{0.33} \mathrm{Ca}_{0.67}$ $\mathrm{RuO}_{3}$, and $\mathrm{CaRuO}_{3}$, respectively. Different microstructures, such as step-like $\left(\mathrm{SrRuO}_{3}, \mathrm{Sr}_{0.33} \mathrm{Ca}_{0.67} \mathrm{RuO}_{3}\right)$ and spiral-like $\left(\mathrm{Sr}_{0.67} \mathrm{Ca}_{0.33} \mathrm{RuO}_{3}\right)$, were observed. More importantly, the grain sizes varied significantly in different buffer layers (larger in $\mathrm{SrRuO}_{3}, \mathrm{Sr}_{0.67} \mathrm{Ca}_{0.33} \mathrm{RuO}_{3}$ and smaller in $\mathrm{Sr}_{0.67}$ $\mathrm{Ca}_{0.33} \mathrm{RuO}_{3}, \mathrm{CaRuO}_{3}$ ) and the larger grain sizes in the buffer layers could facilitate BFO film growth.

Figure 4 shows the I-V curves of the BFO films. The measurement setup is shown in the inset to Figure 4(a). The leakage currents in the $\mathrm{BFO}$ film on $\mathrm{SrRuO}_{3}$ are much smaller than those with other buffer layers. At the maximum electrical field $(8 \mathrm{~V}-210 \mathrm{kV} / \mathrm{cm})$, the leakage current in the film on the $\mathrm{SrRuO}_{3}$-buffer layer was $1.02 \times 10^{3} \mu \mathrm{A} / \mathrm{cm}^{2}$, while for the film on the $\mathrm{Sr}_{0.33} \mathrm{Ca}_{0.67} \mathrm{RuO}_{3}$-buffer layer was $\sim 27.2 \times 10^{3} \mu \mathrm{A} / \mathrm{cm}^{2}$. The maximum leakage currents in different samples are summarized in the inset to Figure 4(c). The polarization reversal currents $\left(\sim 10 \mu \mathrm{A} / \mathrm{cm}^{2}\right)$ contributed little to the measured leakage currents $\left(10^{3}-10^{4} \mu \mathrm{A} / \mathrm{cm}^{2}\right)$. Their effects can be excluded. The sheet resistances of the underlying buffer layers were $9 \Omega / \square, 19 \Omega / \square, 12 \Omega / \square$, and $35 \Omega / \square$ for $\mathrm{SrRuO}_{3}, \mathrm{Sr}_{0.67} \mathrm{Ca}_{0.33} \mathrm{RuO}_{3}, \mathrm{Sr}_{0.33} \mathrm{Ca}_{0.67} \mathrm{RuO}_{3}$, and $\mathrm{CaRuO}_{3}$, respectively. The values are comparable with the published data. ${ }^{26}$ The resistance of the $\mathrm{CaRuO}_{3}$-buffer layer is much larger than the resistance of the other buffer layers, 

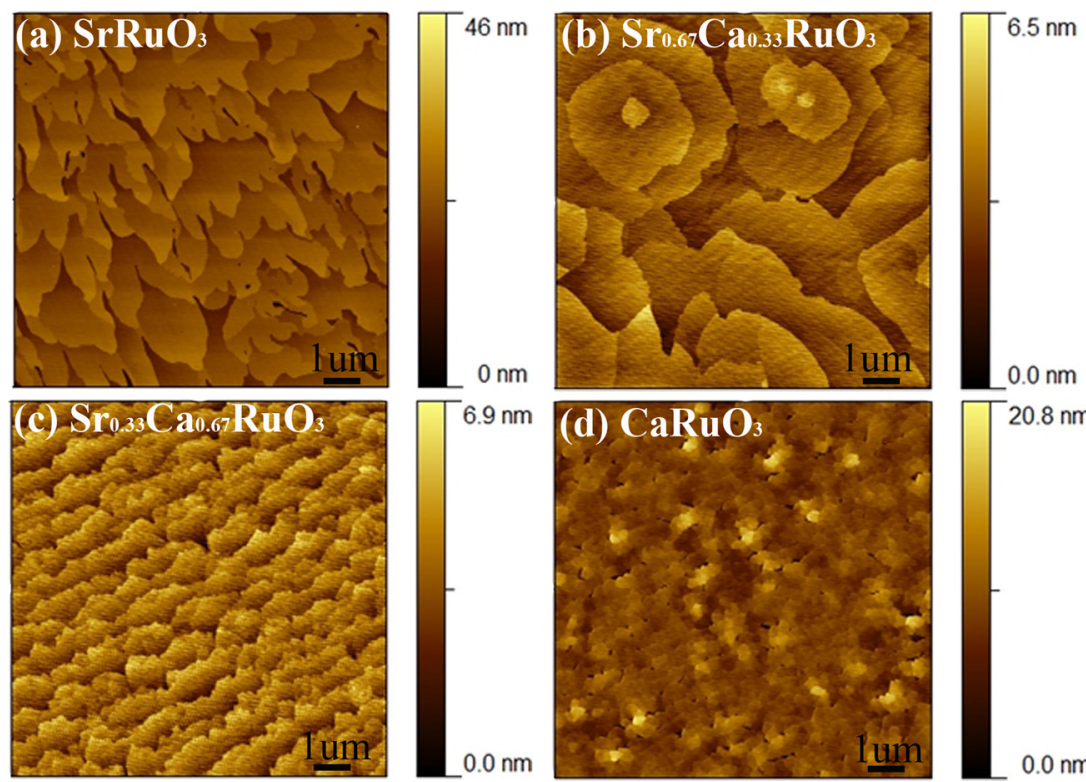

FIG. 3. AFM images of buffer layers (a) $\mathrm{SrRuO}_{3}$, (b) $\mathrm{Sr}_{0.67} \mathrm{Ca}_{0.33} \mathrm{RuO}_{3}$, (c) $\mathrm{Sr}_{0.33} \mathrm{Ca}_{0.67} \mathrm{RuO}_{3}$, and (d) $\mathrm{CaRuO}_{3}$. which could affect the I-V behaviors of the BFO films. Such effects need further investigations.

The most interesting feature of these I-V curves is the highly asymmetric or diode-like I-V behaviors observed in the films grown on $\mathrm{SrRuO}_{3}, \mathrm{Sr}_{0.67} \mathrm{Ca}_{0.33} \mathrm{RuO}_{3}$, and $\mathrm{Sr}_{0.33}$ $\mathrm{Ca}_{0.67} \mathrm{RuO}_{3}$-buffer layers. However, the I-V curves for the film grown on the $\mathrm{CaRuO}_{3}$-buffer layer under positive or negative electrical bias are almost symmetric. Such asymmetric I-V characteristics have been observed in many metalferroelectric-metal and metal-ferroelectric-semiconductormetal configurations. ${ }^{27-36}$ Different top and bottom electrodes may be the origin of these highly asymmetric I-V behaviors, as previously demonstrated in Au-BFO-ZnO, ${ }^{29} \mathrm{Pt}^{-\mathrm{BaTiO}_{3}} \mathrm{3}^{-}$ $\mathrm{ZnO},{ }^{28} \mathrm{Au}-(\mathrm{Pb}, \mathrm{La})\left(\mathrm{Zr}, \mathrm{Ti}^{2} \mathrm{O}_{3}-\mathrm{SrTiO}_{3}: \mathrm{Nb}^{36}{ }^{36}\right.$ Pt-BFO-SRO
(Refs. 37 and 38) heterostructures. In current configurations, i.e., Au-BFO-SCRO, these different bottom and top electrodes can also lead to highly asymmetric I-V behaviors, as two p-n junctions connected back-to-back (as discussed below).

Another intriguing point is the nonconformity of the leakage currents during field-increasing or "forward sweep" and field-decreasing or "reverse sweep" (hysteresis behavior). The nonconformity is considered to be related to ferroelectric polarization states. ${ }^{39}$ Some reports on resistance switching and rectifying diode effects in ferroelectric capacitors suggest that the resistivity of the metal/ferroelectric/metal structure is indeed affected by the polarization states. ${ }^{32,37-41}$ In BFO films, it was found that the BFO-electrode interface barrier could be changed (increased or decreased) by the ferroelectric
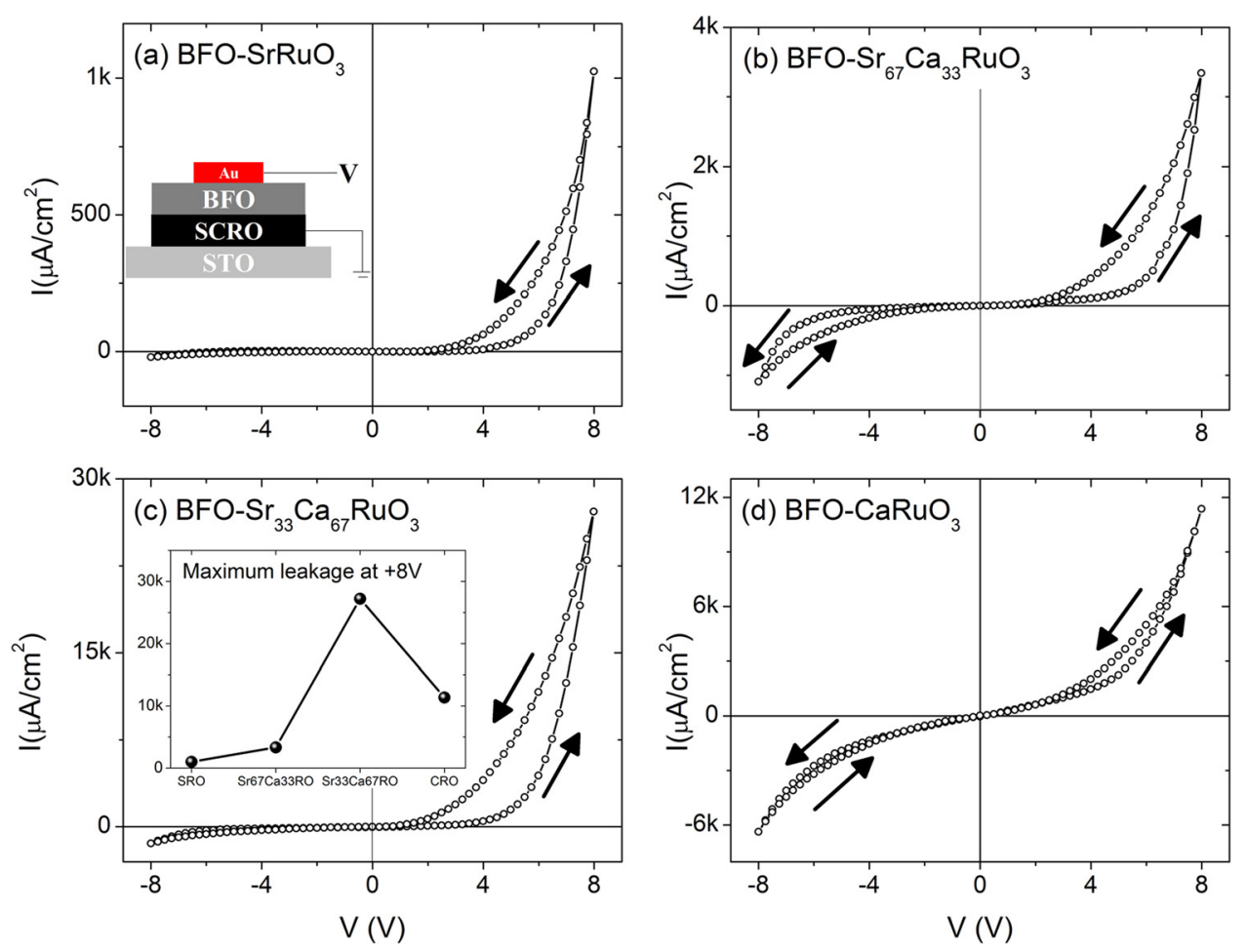

FIG. 4. Leakage currents in BFO thin films on different buffer layers of (a) $\mathrm{SrRuO}_{3}, \quad$ (b) $\mathrm{Sr}_{0.67} \mathrm{Ca}_{0.33} \mathrm{RuO}_{3}, \quad$ (c) $\mathrm{Sr}_{0.33} \mathrm{Ca}_{0.67} \mathrm{RuO}_{3}$, and (d) $\mathrm{CaRuO}_{3}$. The measurement step is $0.2 \mathrm{~V}$ with a delay time of $2 \mathrm{~s}$. The inset in (a) illustrates the I-V measurement configurations. Inset in (c) shows the maximum leakage current at $+8 \mathrm{~V}$ of all films on different buffer layers. 
polarization through the effects of polarization charges. ${ }^{38,41}$ The conduction in BFO films was considered to be p-type, which was induced by Bi-loss (like acceptor doping) during film formation at high temperature. ${ }^{41}$ Under positive bias, the interface between BFO and the bottom SCRO electrode is in the "non-blocking" state (like a forward-biased p-n junction), while the interface between BFO and the top Au electrode is in the "blocking" state (like a reverse-biased p-n junction). Therefore, the leakage current in a SCRO-BFO-Au structure is dominated by the "blocking" BFO-Au interface. Similarly, under negative bias, the leakage is governed by the BFOSCRO interface. Considering that the polarization state under positive bias, during forward sweep $(0 \mathrm{~V} \rightarrow+8 \mathrm{~V})$, the polarization is initially upwards (pointing to top electrode, defined as " $-\mathrm{P}$ ") and then switches to downwards (pointing to bottom electrode, defined as " $+\mathrm{P}$ ") as the applied voltage exceeding a certain point $\left(\mathrm{V}_{\mathrm{c}}\right)$, and stays downwards $(+\mathrm{P})$ during the reverse sweep $(+8 \mathrm{~V} \rightarrow 0)$. Moreover, for downwards polarization $(+\mathrm{P})$, the polarization during fielddecreasing $\left(+8 \mathrm{~V} \rightarrow \mathrm{V}_{\mathrm{c}}\right)$ is larger than that during fieldincreasing $\left(\mathrm{V}_{\mathrm{c}} \rightarrow+8 \mathrm{~V}\right)$ (this holds for a real ferroelectric capacitor; for an ideal one, they are equal). When the polarization direction is $-\mathrm{P}$, positive polarization charges accumulate at the BFO-Au interface, which will increase the potential barrier for the charge carrier (hole) injection from the $\mathrm{Au}$ electrode ("repel holes"). When the polarization switches to $+\mathrm{P}$, negative polarization charges appear at the BFO-Au interface, which will then lower the potential barrier ("attract holes"). The $+\mathrm{P}$ polarization is larger under reverse sweep as stated above, and the potential barrier will be lower. Therefore, the leakage current is larger during the reverse sweep due to the lower interface barrier. The band diagrams under a positive bias with $-\mathrm{P}$ and $+\mathrm{P}$ are shown in Figures 5(a) and 5(b), respectively. The built-in potential barrier can thus be changed by the ferroelectric polarization by, ${ }^{38,41}$

$$
\Delta \emptyset_{b i}=\frac{ \pm p \delta}{\varepsilon_{0} \varepsilon_{s}},
$$

where $\mathrm{P}$ is the ferroelectric polarization, $\delta$ is the thickness of the interface layer between the surface polarization charge and the electrode, $\varepsilon_{0}$ is the vacuum permittivity, and $\varepsilon_{\mathrm{s}}$ is the static dielectric constant of the film. Wang et al. obtained a $\Delta \emptyset_{b i}$ of $1.38 \mathrm{eV}$ using $\mathrm{P}=60 \mu \mathrm{C} / \mathrm{cm}^{2}, \delta=1 \mathrm{~nm}, \varepsilon_{\mathrm{s}}=50$, for a $240 \mathrm{~nm}$ BFO film in a Pt-BFO-SRO structure prepared by $\mathrm{PLD},{ }^{37}$ while Lee et al. calculated $\Delta \emptyset_{b i}$ to be $0.6 \mathrm{eV}$ using $\mathrm{P}$ $=65 \mu \mathrm{C} / \mathrm{cm}^{2}, \delta=1 \mathrm{~nm}, \varepsilon_{\mathrm{s}}=100$, for a $400 \mathrm{~nm}$ BFO film in a Pt-BFO-SRO structure deposited by sputtering. ${ }^{38}$ In our case, $\mathrm{P}$ is only $15.5 \mu \mathrm{C} / \mathrm{cm}^{2}$ for the Au-BFO-SRO sample. The change in the potential barrier would therefore be expected to be $0.1-0.4 \mathrm{eV}$. The potential barrier height without charges at the interface is expected to be $\sim 0.6 \mathrm{eV}$, which depends on the density of impurities in the film, especially around the interface region. ${ }^{41}$ Next, we consider the case of negative biases, where the interface between BFO and $\mathrm{Au}$ is "non-blocking" and that between BFO and SCRO is "blocking." In the same way, the leakage current during forward sweep $(0 \rightarrow-8 \mathrm{~V})$ is lower than that during reverse sweep $(-8 \mathrm{~V} \rightarrow 0)$. However, compared with the case of positive biases, suppressed hysteresis and much lower leakage currents are observed, which indicates a higher potential barrier at the BFO-SCRO interface under negative bias than that at the BFO-Au interface under positive bias. The higher barrier may be induced by the accumulated positively charged oxygen vacancies $\left(\mathrm{V}_{\mathrm{o}}\right)$ at the BFO-SCRO interface due to the self-poled upwards polarization (parallel with $-\mathrm{P})$ : a negative polarization charge is built up near the bottom SCRO electrode, which attracts $V_{0}$ to migrate towards the SCRO electrode at high temperatures during deposition. ${ }^{41}$ A rough interface may facilitate the accumulation of these oxygen vacancies. The roughness of the SRO bottom

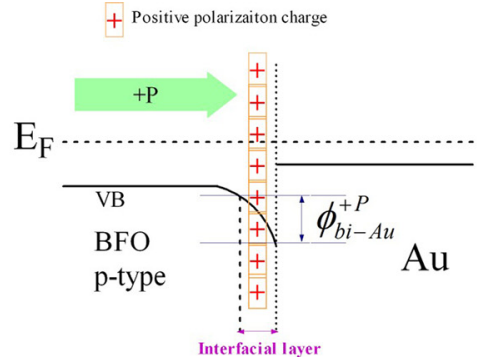

(a) Positive forward bias $\left(0 \rightarrow+V_{c}\right)$

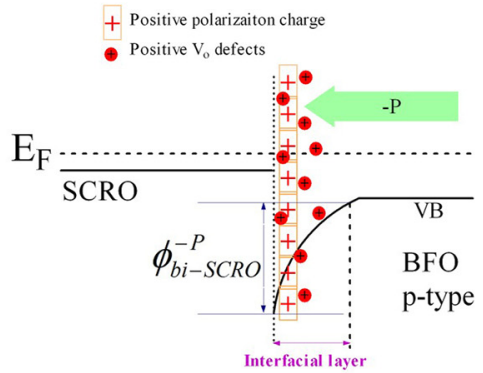

(c) Negative forward bias $\left(0 \rightarrow-V_{c}\right)$

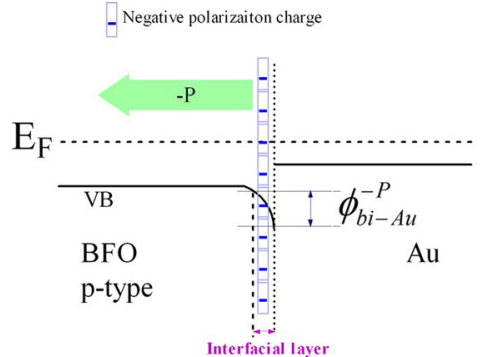

(b) Positive reverse bias $\left(+V_{\max } \rightarrow 0\right)$

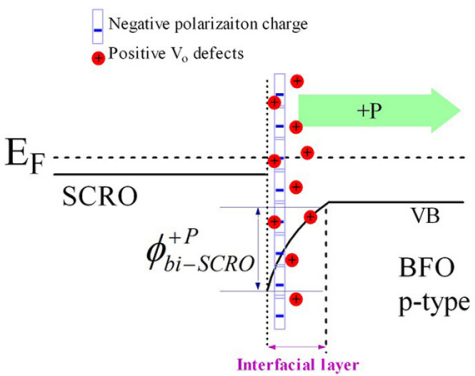

(d) Negative reverse bias $\left(-\mathrm{V}_{\max } \rightarrow 0\right)$
FIG. 5. Band diagrams for the BFO-Au interface under positive (a) forward, (b) reverse bias and the BFOSCRO interface under negative (c) forward, and (d) reverse bias. The applied field (positive or negative) is at the top Au electrode. $\mathrm{E}_{\mathrm{F}}$ : Fermi level, VB: valence band, $\mathrm{V}_{\mathrm{o}}$ : positively charged oxygen vacancies, $\varnothing_{b i-A u}^{+P}$ : built-in potential barrier at the BFO-Au interface with the ferroelectric polarization inside the film pointing to the top Au electrode $(-\mathrm{P}), \varnothing_{b i-A u}^{-P}$ : built-in potential barrier at the $\mathrm{BFO}-\mathrm{Au}$ interface with the ferroelectric polarization inside the film pointing to the bottom SCRO electrode $(+\mathrm{P}), \varnothing_{b i-S C R O}^{+P}$ : built-in potential barrier at the BFO-SCRO interface with the ferroelectric polarization inside the film pointing to the top Au electrode $(-\mathrm{P}), \phi_{b i-S C R O}^{-P}$ : built-in potential barrier at the BFO-SCRO interface with the ferroelectric polarization inside the film pointing to the bottom SCRO electrode $(+\mathrm{P})$. 

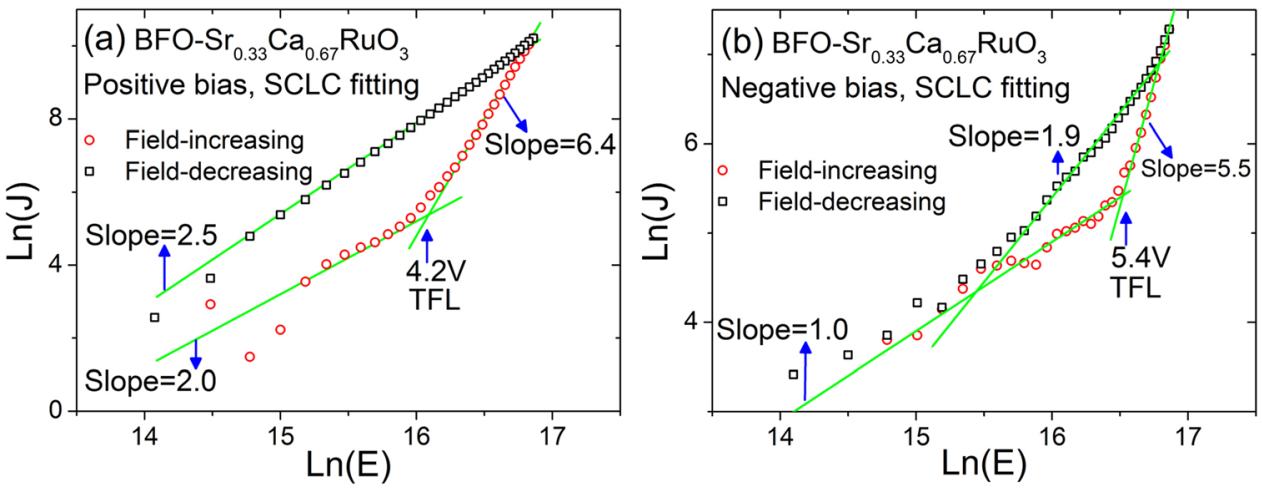

FIG. 6. Fitting results for the leakage currents in $\mathrm{BFO}$ films on the $\mathrm{Sr}_{0.33} \mathrm{Ca}_{0.67}$ $\mathrm{RuO}_{3}$-buffer layers under (a) positive and (b) negative bias. "TFL" indicates the onset of the traps-filled-level. electrode $(1.79 \mathrm{~nm})$ is the greatest among all the SCRO electrodes and the film on the SRO electrodes exhibits the most striking asymmetric I-V behavior. These localized permanent positive charges lead to the formation of a high potential barrier at the SCRO-BFO interface for the positive charge carriers (holes), as shown in Figures 5(c) and 5(d). The barrier height is so large that it overtakes the effects of the ferroelectric polarization charges. Therefore, the leakage current under negative bias is much smaller and the hysteresis is also not that exaggerated. This scenario also accounts for the asymmetric I-V behaviors.

Several types of leakage or conduction mechanisms may contribute the leakage current, including Ohmic conduction, space-charge-limited-conduction (SCLC), interface-limited Schottky emission, bulk-limited Poole-Frenkel (PF) emission, interface-limited Fowler-Nordheim (FN) tunneling, and hopping conduction (HC). ${ }^{27-35}$ These mechanisms can be described by the following equations,

$$
J_{S C L C}=\frac{9 \mu \varepsilon_{0} \varepsilon_{r}}{8 d} E^{n}
$$

where $\mathrm{d}$ is the film thickness, $\mu$ is the charger carrier mobility, $\varepsilon_{0}$ is the vacuum permittivity, $\varepsilon_{\mathrm{r}}$ is the relative dielectric constant of the film, $\mathrm{E}$ is the electrical field, $\mathrm{J}$ is the current density, $\mathrm{n}=2$ for trap-free conditions, $\mathrm{n}>2$ for SCLC with charge carrier traps;

$$
J_{\text {Schottky }}=A T^{2} e^{\frac{-\emptyset-q \sqrt{q E / \pi \varepsilon_{0} K}}{k_{B} T}}
$$

where A is constant, $\varphi$ is the Schottky barrier, $\mathrm{T}$ is the absolute temperature in Kelvin, $\mathrm{q}$ is the elemental charge, $\mathrm{K}$ is the optical dielectric permittivity ( $\mathrm{K} \sim 6.25$ for $\mathrm{BFO}$ ), $\mathrm{k}_{\mathrm{B}}$ is the Boltzmann constant;

$$
J_{P F}=B E e^{\frac{-E_{I}-q \sqrt{q E / \pi \varepsilon_{0} K}}{k_{B} T}}
$$

where $\mathrm{B}$ is constant and $\mathrm{E}_{\mathrm{I}}$ is the trap ionization energy;

$$
J_{F N}=C E^{2} e^{\frac{-D^{2} \sqrt{\varphi_{i}^{E}}}{E}}
$$

where $\mathrm{C}$ and $\mathrm{D}$ are constants and $\varphi_{\mathrm{i}}$ is the potential barrier height;

$$
J_{\text {Hopping }}=q a n v e^{\frac{q a E}{k_{B} T}-\frac{E_{a}}{k_{B} T}}
$$

where $\mathrm{a}$ is the average hopping distance, $\mathrm{n}$ is the charge carrier density, $v$ is the frequency of the thermal vibration of the electrons at the trap sites, and $\mathrm{E}_{\mathrm{a}}$ is the activation energy of trapped charges.

By fitting the experimental data in the coordinates of $\ln (\mathrm{J})$ vs. $\ln (\mathrm{E})$ (SCLC or Ohmic), J/T $\mathrm{T}^{2}$ vs. $\mathrm{E}^{0.5}$ (Schottky), J/E vs. $\mathrm{E}^{0.5}(\mathrm{PF}), \mathrm{J} / \mathrm{E}^{2}$ vs. $\mathrm{E}^{-1}(\mathrm{FN})$, and $\ln (\mathrm{J})$ vs. E (Hopping), it is possible to determine which mechanisms contribute to the leakage currents. In all BFO films, SCLC dominated the leakage currents under a high electrical field and Ohmic conduction under a low electric field. The conduction mechanisms under a negative bias resembled those under a positive bias. The slope $(n)$ of the $\operatorname{Ln}(J)-\operatorname{Ln}(E)$ plot in the SCLC region was found to be 1.7-3.0, deviating from the ideal value of 2 . This is related to the distribution of the charge carrier traps in the bandgap, which depends on the applied field. ${ }^{34}$ One example of these fittings is shown in Figure 6 for the BFO on $\mathrm{Sr}_{0.33} \mathrm{Ca}_{0.67} \mathrm{RuO}_{3}$. The filled traps level was also observed, where $\mathrm{n}$ was much larger than 2 (6.4 and 5.5 as shown in Figure 6). The physical nature of the charge carrier traps is believed to be the structural defects or impurities, for example the cation/anion vacancies. ${ }^{42}$

The ferroelectric hysteresis loops of the films are shown in Figure 7. All films exhibited well-defined loops. However, the films grown on $\mathrm{Sr}_{0.33} \mathrm{Ca}_{0.67} \mathrm{RuO}_{3}$-buffer layer exhibited a rounded shape, indicating considerable contribution from leakage currents. The remnant polarization $\left(P_{r}\right)$ and coercive field $\left(E_{c}\right)$ in the film on the $\mathrm{SrRuO}_{3}$ buffer layer are $15.5 \mu \mathrm{C} / \mathrm{cm}^{2}$ and $176 \mathrm{kV} / \mathrm{cm}$, respectively.

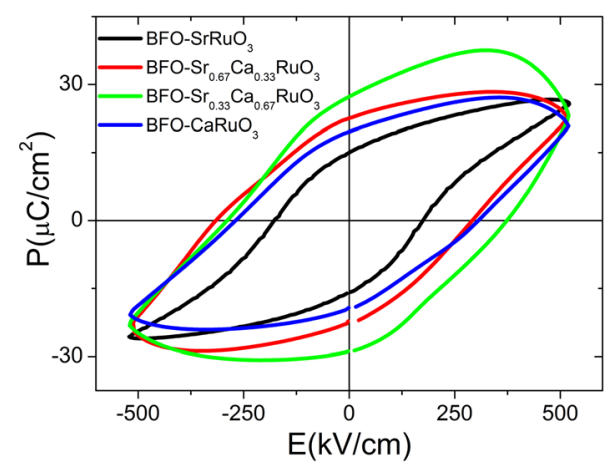

FIG. 7. Ferroelectric hysteresis loops of BFO thin films on $\mathrm{Sr}_{\mathrm{x}} \mathrm{Ca}_{1-\mathrm{x}} \mathrm{RuO}_{3^{-}}$ buffered $\mathrm{SrTiO}_{3}$ substrates. 


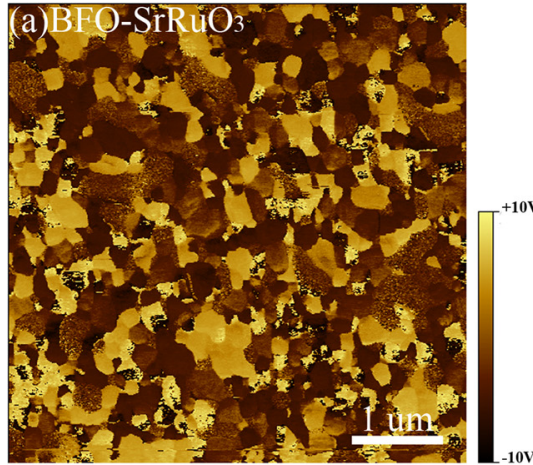

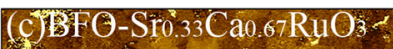

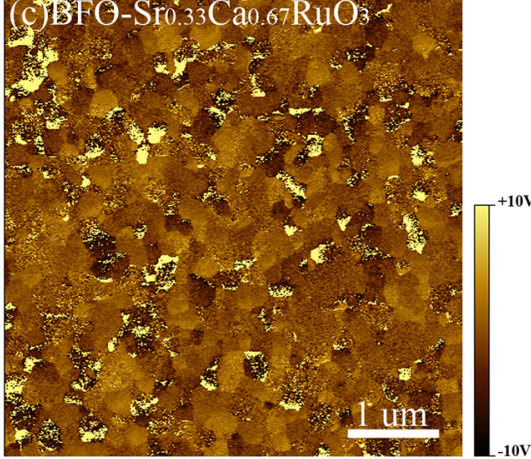

The small $\mathrm{P}_{\mathrm{r}}$, compared with other BFO films ( 50-130 $\mu \mathrm{C} / \mathrm{cm}^{2}$ (Refs. 1, 5, 6, and 9)), may be due to the secondary phase or smaller grain size in our samples, as shown in Figure 1(a).
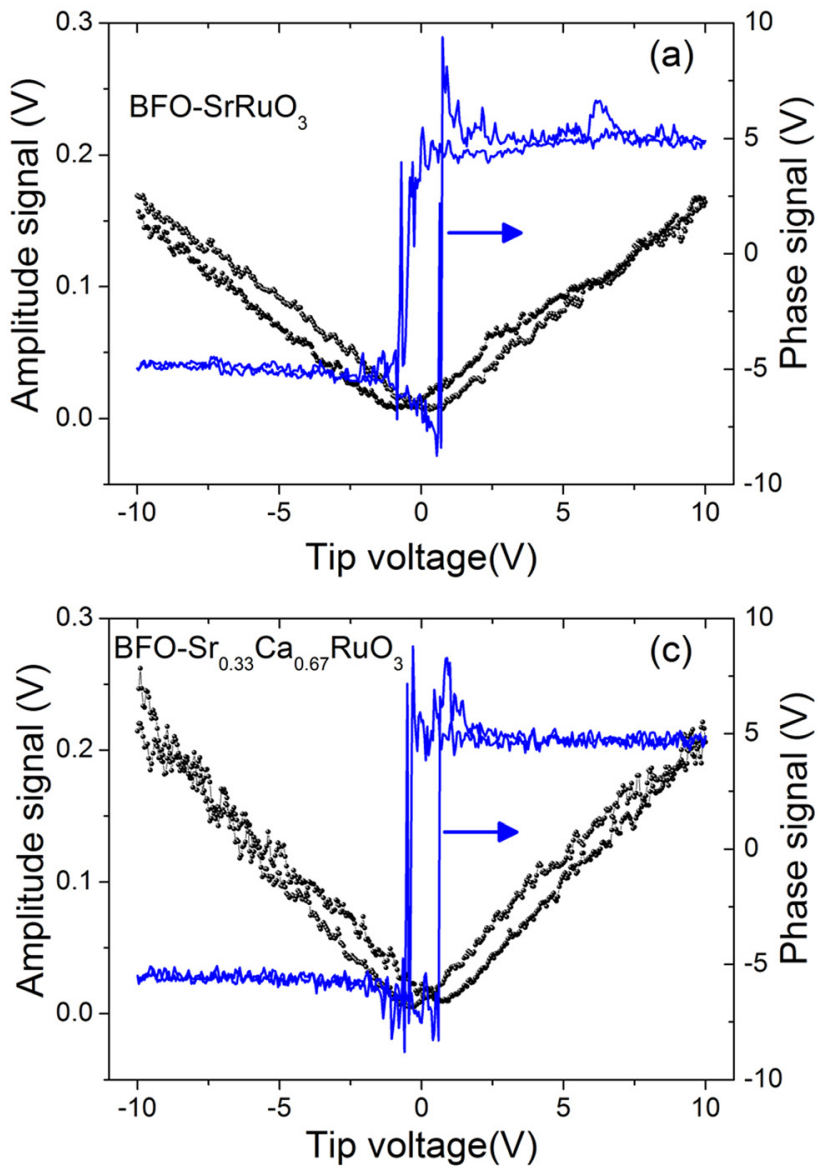

The piezoelectric responses of the films are shown in Figures 8 and 9. Figure 8 shows the piezoelectric force microscopy (PFM) phase pictures of non-poled films. Large ferroelectric domains are clearly observed in the BFO films
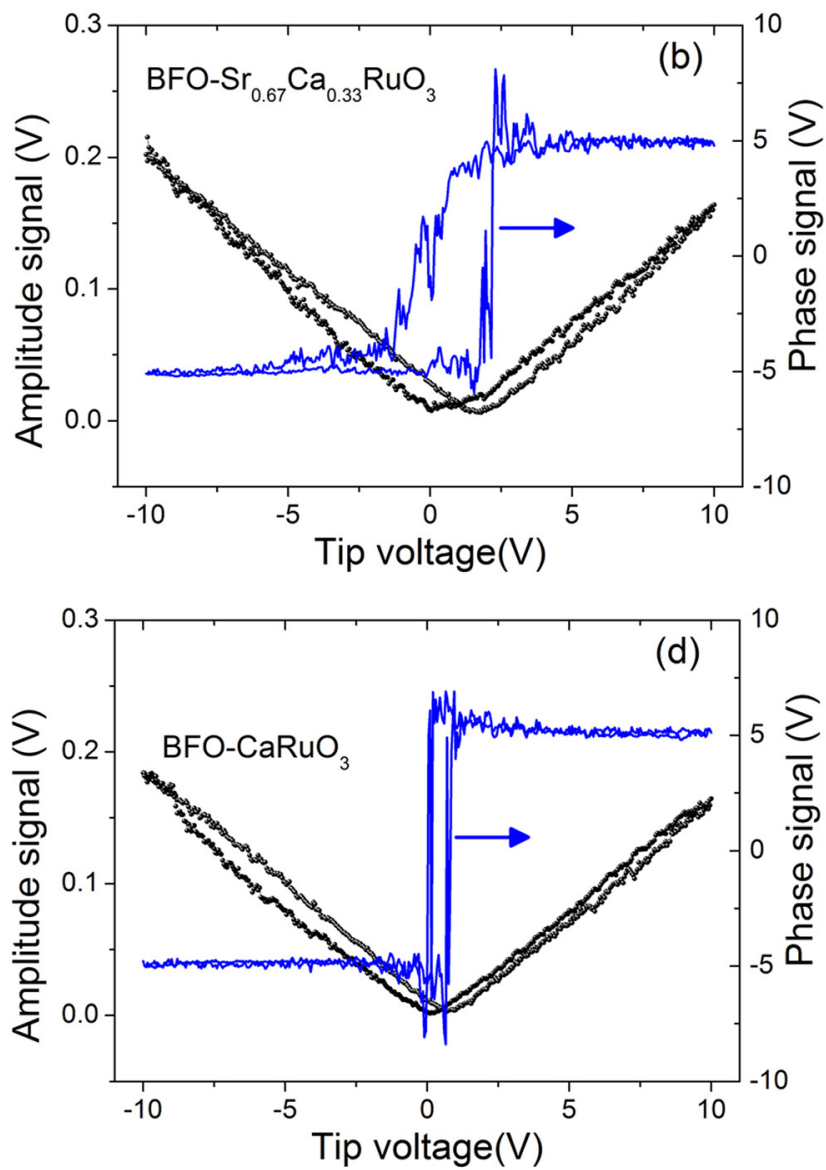

FIG. 9. Local piezo-responses, amplitudes (black solid circles) and phases (blue solid lines), of (a) $\mathrm{BFO}_{-} \mathrm{SrRuO}_{3}$, (b) $\mathrm{BFO}_{-} \mathrm{Sr}_{0.67} \mathrm{Ca} \mathrm{a}_{0.33} \mathrm{RuO}_{3}$, (c) $\mathrm{BFO}$ $\mathrm{Sr}_{0.33} \mathrm{Ca}_{0.67} \mathrm{RuO}_{3}$, and (d) $\mathrm{BFO}-\mathrm{CaRuO}_{3}$. 
grown on $\mathrm{SrRuO}_{3}$ and $\mathrm{Sr}_{0.67} \mathrm{Ca}_{0.33} \mathrm{RuO}_{3}$-buffer layers. The domain sizes are much smaller and the contrast between different domains is blurred in these two samples. Two factors may account for these differences: the film orientation and grain size. As shown previously, the films grown on $\mathrm{SrRuO}_{3}$ and $\mathrm{Sr}_{0.67} \mathrm{Ca}_{0.33} \mathrm{RuO}_{3}$-buffer layers are highly (001) oriented and exhibit larger grain sizes $(260 \mathrm{~nm}$ and $207 \mathrm{~nm}$ ). The (110) orientation was observed in the other two samples, even at a low percentage and the grain sizes are also smaller $(186 \mathrm{~nm}$ and $134 \mathrm{~nm}$ ). However, the piezo-response (amplitude) is the largest in the film grown on the $\mathrm{Sr}_{0.33} \mathrm{Ca}_{0.67} \mathrm{RuO}_{3}$-buffer layer, which is $0.233 \mathrm{~V}$ under an applied field of $10 \mathrm{~V}$. The corresponding values for the films on $\mathrm{SrRuO}_{3}, \mathrm{Sr}_{0.67} \mathrm{Ca}_{0.33} \mathrm{RuO}_{3}$, and $\mathrm{CaRuO}_{3}$-buffer layers are $0.167 \mathrm{~V}, 0.184 \mathrm{~V}$, and $0.172 \mathrm{~V}$, respectively, as shown in Figure 8. Another interesting point observed in Figure 9 is the smaller local coercive fields in the sample grown on $\mathrm{Sr}_{0.33} \mathrm{Ca}_{0.67} \mathrm{RuO}_{3}$ and $\mathrm{CaRuO}_{3}$-buffer layers, which are $0.49 \mathrm{~V}$ and $0.40 \mathrm{~V}$ compared with $0.62 \mathrm{~V}$ in BFO$\mathrm{SrRuO}_{3}$ and $1.21 \mathrm{~V}$ in $\mathrm{BFO}-\mathrm{Sr}_{0.67} \mathrm{Ca}_{0.33} \mathrm{RuO}_{3}$. This difference may be related to the larger lattice-mismatch strains in the films on $\mathrm{Sr}_{0.33} \mathrm{Ca}_{0.67} \mathrm{RuO}_{3}$ and $\mathrm{CaRuO}_{3}$ buffer layers, as discussed previously. The large strain will deform the crystal structure of BFO from rhombohedral to tetragonal to some extent and then improve the piezoelectric $\left(\mathrm{d}_{33}\right)$ and ferroelectric $\left(\mathrm{P}_{\mathrm{r}}\right.$ and $\left.\mathrm{E}_{\mathrm{c}}\right)$ properties. ${ }^{12,13,19}$ In our case, the structural differences cannot be determined as their XRD diffraction peaks are overshadowed by the buffer layers. But the lattice constants of the films with different buffer layers are indeed different, as shown in the XRD results. Therefore, we may expect lattice deformation in films with larger strains and therefore enhanced piezoelectric performance. The final point worth mentioning is the much smaller coercive fields $(0.40-1.21 \mathrm{~V})$ measured by local PFM compared with those (6.69-12.49 V, see Figure 7) obtained from the ferroelectric hysteresis measurements. The origin for this may be (1) the smaller electrode area ( $\sim 20 \mathrm{~nm}$ in diameter) of the PFM tip, which could induce enhancement of a localized electrical field due to the sharpness of the tip and the tip-contact geometry ${ }^{27}$ and (2) the non-ferroelectric secondary phases (as shown in Figure 1(a)), which will hinder the polarization reversal in larger area $\left(2 \times 10^{5} \mathrm{~nm}\right.$ in diameter) measurements and may not manifest itself in the local $(20 \mathrm{~nm}$ in diameter) PFM measurements.

\section{CONCLUSIONS}

Effects of buffer layers $\left(\mathrm{SrRuO}_{3}, \mathrm{Sr}_{0.67} \mathrm{Ca}_{0.33} \mathrm{RuO}_{3}\right.$, $\mathrm{Sr}_{0.33} \mathrm{Ca}_{0.67} \mathrm{RuO}_{3}, \mathrm{CaRuO}_{3}$ ) on the microstructural, crystallographic, electrical, ferroelectric, and piezoelectric properties of (001)-oriented BFO thin films were systematically studied. It was found that the lattice constants of the BFO films changed gradually as the buffer layer was varied from $\mathrm{SrRuO}_{3}$ to $\mathrm{CaRuO}_{3}$. Diode behavior in the I-V curves was observed in the BFO films on $\mathrm{SrRuO}_{3}, \mathrm{Sr}_{0.67} \mathrm{Ca}_{0.33} \mathrm{RuO}_{3}$, $\mathrm{Sr}_{0.33} \mathrm{Ca}_{0.67} \mathrm{RuO}_{3}$-buffered layers. Space-charge-limited-conduction mechanism was verified in the leakage of all the films. The film on $\mathrm{SrRuO}_{3}$ exhibited the lowest leakage current and a well-saturated ferroelectric hysteresis loop. Different piezoelectric responses, revealed by PFM method, were observed in these films. BFO films with large strains showed increased piezo responses. Different strain states in these films may account for these observations.

\section{ACKNOWLEDGMENTS}

We thank Mr. Xiaodong Xu from the Mechanical Workshop at KAUST for preparation of the sample's fixture on our PLD system.

${ }^{1}$ J. Wang et al., Science 299, 1719 (2003).

${ }^{2}$ A. Stroppa and S. Picozzi, Phys. Chem. Chem. Phys. 12, 5405 (2010).

${ }^{3}$ S. Y. Yang, J. Seidel, S. J. Byrnes, P. Shafer, C. H. Yang, M. D. Rossell, P. Yu, Y. H. Chu, J. F. Scott, J. W. Ager III, L. W. Martin, and R. Ramesh, Nat. Nanotechnol. 5, 143 (2010).

${ }^{4}$ S. Y. Yang, L. W. Martin, S. J. Byrnes, T. E. Conry, S. R. Basu, D. Paran, L. Reichertz, J. Ihlefeld, C. Adamo, A. Melville, Y. H. Chu, C. H. Yang, J. L. Musfeldt, D. G. Schlom, J. W. Ager III, and R. Ramesh, Appl. Phys. Lett. 95, 062909 (2009).

${ }^{5}$ W. Ji, K. Yao, and Y. C. Liang, Adv. Mater. 22, 1763 (2010).

${ }^{6}$ R. K. Katiyar, A. Kumar, G. Morell, J. F. Scott, and R. S. Katiyar, Appl. Phys. Lett. 99, 092906 (2011).

${ }^{7}$ G. Catalan, J. Seidel, R. Ramesh, and J. F. Scott, Rev. Mod. Phys. 84, 119 (2012).

${ }^{8}$ G. L. Yuan, S. W. Or, Y. P. Wang, Z. G. Liu, and J. M. Liu, Solid State Commun. 138, 76 (2006).

${ }^{9}$ I. Sosnowska, M. Loewenhaupt, W. I. F. David, and R. M. Ibberson, Physica B 180-181, 117 (1992).

${ }^{10}$ V. R. Palkar, D. C. Kundaliya, S. K. Malik, and S. Bhattacharya, Phys. Rev. B 69, 212102 (2004).

${ }^{11}$ T.-J. Park, G. C. Papaefthymiou, A. J. Viescas, A. R. Moodenbaugh, and S. S. Wong, Nano Lett. 7, 766 (2007).

${ }^{12}$ J. X. Zhang et al., Phys. Rev. Lett. 107, 147602 (2011).

${ }^{13}$ J. X. Zhang et al., Nat. Nanotechnol. 6, 98 (2011).

${ }^{14}$ R. K. Vasudevan, Y. Liu, J. Li, W.-I. Liang, A. Kumar, S. Jesse, Y.-C. Chen, Y.-H. Chu, V. Nagarajan, and S. V. Kalinin, Nano Lett. 11, 3346 (2011).

${ }^{15}$ J. Kreisel, P. Jadhav, O. Chaix-Pluchery, M. Varela, N. Dix, F. Sánchez, and J. Fontcuberta, J. Phys.: Condens. Matter 23, 342202 (2011).

${ }^{16}$ C. B. Eom et al., Science 258, 1766 (1992).

${ }^{17}$ X. G. Tang, H. Y. Tian, J. Wang, K. H. Wong, and H. L. W. Chan, Appl. Phys. Lett. 89, 142911 (2006).

${ }^{18}$ R. J. Zeches et al., Science 326, 977 (2009).

${ }^{19}$ H. W. Jang et al., Phys. Rev. Lett. 101, 107602 (2008).

${ }^{20}$ S. K. Singh, N. Menou, H. Funakubo, K. Maruyama, and H. Ishiwara, Appl. Phys. Lett. 90, 242914 (2007).

${ }^{21}$ Y.-H. Lee, C.-S. Liang, and J.-M. Wu, Electrochem. Solid-State Lett. 8(11), F55 (2005).

${ }^{22}$ S. M. Selbach, M.-A. Einarsrud, and T. Grande, Chem. Mater. 21, 169 (2009).

${ }^{23}$ M. S. Bernardo, T. Jardiel, M. Peiteado, A. C. Caballero, and M. Villegas, J. Eur. Ceram. Soc. 31, 3047 (2011).

${ }^{24}$ H. O. Rodrigues, G. F. M. Pires, Jr., J. S. Almeida, E. O. Sancho, A. C. Ferreira, M. A. S. Silva, and A. S. B. Sombra, J. Phys. Chem. Solids 71, 1329 (2010).

${ }^{25}$ P. Chaudhari, J. Vac. Sci. Technol. 9, 520 (1971).

${ }^{26}$ H.-C. Lee and D.-S. Tsai, J. Mater. Sci. 38, 2633 (2003); K. Gurunathan, N. Vyawahare, and D. P. Amalnerkar, J. Mater. Sci.: Materials in Electronics 16, 47 (2005).

${ }^{27}$ P. Maksymovych, M. Pan, P. Yu, R. Ramesh, A. P. Baddorf, and S. V. Kalinin, Nanotechnology 22, 254031 (2011); P. Maksymovych, S. Jesse, P. Yu, R. Ramesh, A. P. Baddorf, and S. V. Kalinin, Science 324, 1421 (2009).

${ }^{28}$ V. M. Voora, T. Hofmann, M. Brandt, M. Lorenz, M. Grundmann, N. Ashkenov, and M. Schubert, Appl. Phys. Lett. 94, 142904 (2009).

${ }^{29}$ J. Wu and J. Wang, J. Appl. Phys. 108, 034102 (2010).

${ }^{30}$ J. Wu, J. Wang, D. Xiao, and J. Zhu, AIP Adv. 1, 022138 (2011).

${ }^{31}$ G. W. Pabst, L. W. Martin, Y.-H. Chu, and R. Ramesh, Appl. Phys. Lett. 90, 072902 (2007).

${ }^{32}$ H. Yang, M. Jain, N. A. Suvorova, H. Zhou, H. M. Luo, D. M. Feldmann, P. C. Dowden, R. F. DePaula, S. R. Foltyn, and Q. X. Jia, Appl. Phys. Lett. 91, 072911 (2007).

${ }^{33}$ T. Choi, S. Lee, Y. J. Choi, V. Kiryukhin, and S. W. Cheong, Science 324, 63 (2009). 
${ }^{34}$ F.-C. Chiu, C.-Y. Lee, and T.-M. Pan, J. Appl. Phys. 105, 074103 (2009).

${ }^{35}$ A. J. Campbell, D. D. C. Bradley, and D. G. Lidzey, J. Appl. Phys. 82, 6326 (1997).

${ }^{36}$ Y. Watanabe, Phys. Rev. B 59, 11257 (1999).

${ }^{37}$ C. H. Ahn et al., Science 276, 1100 (1997); C. H. Ahn et al., Appl. Phys. Lett. 70, 206 (1997); E. M. Bourim, S. Park, X. Liu, K. P. Biju, H. Hwang, and A. Ignatiev, Electrochem. Solid-State Lett. 14(5), H225 (2011).
${ }^{38}$ C. Wang, K.-J. Jin, Z.-T. Xu, L. Wang, C. Ge, H.-B. Lu, H.-Z. Guo, M. He, and G.-Z. Yang, Appl. Phys. Lett. 98, 192901 (2011).

${ }^{39}$ Y. Watanabe, Appl. Phys. Lett. 66, 28 (1995).

${ }^{40}$ A. Beck, J. G. Bednorz, Ch. Gerber, C. Rossel, and D. Widmer, Appl. Phys. Lett. 77, 139 (2000)

${ }^{41}$ D. Lee, S. H. Baek, T. H. Kim, J. G. Yoon, C. M. Folkman, C. B. Eom, and T. W. Noh, Phys. Rev. B 84, 125305 (2011).

${ }^{42}$ E. A. Silinsh, Phys. Status Solidi A 3, 817 (1970). 